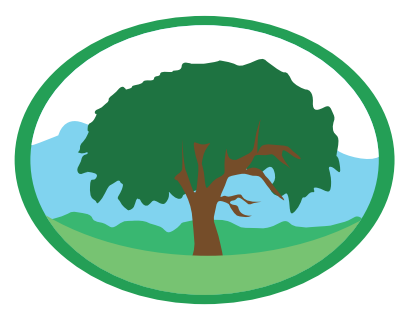

\title{
CURVAS DE INTENSIDADE-DURAÇÃO-FREQUÊNCIA: UM MAPEAMENTO SISTEMÁTICO
}

SANTOS NETO, E. P.; SARMENTO, A. P.

Palavras-chave:

gumbel, kolmogorov-smirnov, algoritmos heurísticos.

\section{chuva, Resumo}

A curva de intensidade, duração e frequência (curva IDF) é o método utilizado para se obter a intensidade máxima de precipitação associada a uma determinada duração e a um período de retorno, e este valor é de extrema importância para o cálculo da vazão máxima de projeto, a qual é necessária para dimensionamento de vertedouros, canais, galerias pluviais, bueiros, sistemas de drenagem e de irrigação. Logo, o presente trabalho tem por objetivo apresentar uma revisão sistematizada sobre o cálculo dos parâmetros da curva de intensidade, duração e frequência através do mapeamento sistêmico, tomando como fonte de pesquisa a base de dados CAPES. Os resultados obtidos mostraram que a distribuição probabilística de Gumbel e o teste de aderência de Kolmogorov-Smirnov foram os mais utilizados nos artigos e que ocorreu um crescimento no número de pesquisas principalmente nos últimos 10 anos. No entanto, verificou-se que uma pequena parcela destas aplicaram algoritmos heurísticos na determinação dos parâmetros da curva IDF.

\section{CURVES OF INTENSITY-DURATION-FREQUENCY: A MAPPING STUDY}

Keywords: rainfall, Gumbel, Kolmogorov-Smirnov, heuristic algorithms.

\begin{abstract}
The intensity, duration and frequency curve (IDF curve) is the method used to obtain the maximum intensity of precipitation associated with a given duration and a return period, and this value is extremely important for calculating the maximum design flow, which is required for sizing of spillways, canals, rain galleries, drains and irrigation systems. Therefore, this paper aims to present a systematic review on the calculation of the parameters of the intensity, duration and frequency curve through systemic mapping, taking as a source of research the CAPES database. The results obtained showed that the probabilistic distribution of Gumbel and the Kolmogorov-Smirnov adherence test were the most used in the articles and that there has been an increase in the number of researches mainly in the last 10 years. However, it was found that a small portion of these applied heuristic algorithms in determining the parameters of the IDF curve.
\end{abstract}




\section{INTRODUÇÃO}

Embora os fenômenos hidrológicos como as chuvas e o escoamento dos rios possam parecer comuns e muito conhecidos, devido à sua irregularidade ao longo do tempo e do espaço, eles podem vir a causar efeitos catastróficos provocando danos materiais e a perda de vidas (PINTO et al., 1976; ALHASSOUN, 2011). Dessa maneira, o conhecimento das características dos fenômenos hidrológicos é fundamental para proporcionar a elaboração de projetos relacionados à utilização dos recursos hidráulicos de forma mais segura, fazendo com que seu estudo deixe de ser uma ciência apenas estudada no âmbito acadêmico para se tornar uma ferramenta imprescindível ao engenheiro (PINTO et al., 1976; SILVA; CÂNDIDO; PIRES; FRANÇA, 2018).

Dentre esses projetos pode-se citar o dimensionamento de drenos, vertedores, obras de proteção contra cheias, barragens, canais, desvios de cursos d'água, galerias pluviais, bueiros, sistemas de drenagem e de irrigação, controle da erosão hídrica do solo, dentre outros (CARDOSO; ULLMANN; BERTOL, 1998; BORGES; THEBALDI, 2016; SILVA; CÂNDIDO; PIRES; FRANÇA, 2018).

Para se dimensionar essas estruturas faz-se necessário conhecer a vazão máxima de projeto, que devido escassez de dados fluviométricos acaba sendo determinada a partir de dados pluviométricos através da intensidade máxima de precipitação, obtida por meio da curva de intensidade, duração e frequência (curva IDF), utilizando-se a metodologia de desagregaçáo de chuvas, da CETESB (1980).

Tendo em vista que essa metodologia engloba uma série de etapas, o presente artigo tem como objetivo apresentar um mapeamento sistêmico, apoiando-se na base de dados CAPES, acerca dos estudos relacionados às curvas IDF, buscando apontar as principais distribuiçôes probabilísticas teóricas utilizadas, os principais modelos de equaçóes IDF, métodos de otimizaçáo, testes de aderência e coeficientes para avaliação do ajuste de parâmetros.

\section{MATERIAL E MÉTODOS}

\section{Mapeamento sistemático}

O Mapeamento Sistemático, também conhecido como Systematic mapping, é usado para fornecer uma visão geral de uma área de pesquisa, para estabelecer se a evidência de pesquisa existe e para fornecer uma indicação da quantidade de evidência (KITCHENHAM; CHARTERS, 2007). Ele consiste em uma busca por literaturas em bancos de dados, através de operadores lógicos (strings) estabelecidos, cujo objetivo é selecionar referências e obter um panorama sobre as pesquisas referentes a um determinado assunto (COSTA; STAUT; ILHA, 2014).

O procedimento baseia-se nas seguintes etapas: definição dos bancos de dados de busca, determinação das strings, aplicação dos filtros (área de estudo, período, idioma etc.), aderência das referências a partir do título, aderência das referências a partir do resumo, snowball sampling (revisão das bibliografias utilizadas nas referências aderentes, a fim de se obter outras referências não selecionadas no mapeamento), exclusáo dos artigos repetidos dentro das buscas, análise dos artigos selecionados e apresentação dos resultados obtidos.

\section{Coleta de periódicos na plataforma}

Nesta pesquisa, as buscas foram realizadas no banco de dados CAPES, disponível através do servidor da Universidade Federal de Goiás - Regional Cataláo. Os operadores lógicos e as strings adotadas foram: combinação 1 (C1), "Intensity-duration-frequency" e "Estimt*"; combinação 2 (C2), "Intensity-durationfrequency" e "Optimization"; combinação 3 (C3), "Intensity-duration- frequency" e "Algorithm". Após a busca inicial, foram adotados como filtros: o idioma (inglês, espanhol e português), o tipo de periódico (artigos) e o tipo de publicação (artigo de periódico revisado por pares).

Não foram aplicados filtros em relação à "área de conhecimento", tais como Engenharia, Ciências da Terra, Geografia, no entanto foram aplicados filtros com relação ao tópico para cada combinação, a saber: (C1) environmental sciences, storms, climate, runoff, mathematical models, floods, geography, statistical analysis, engineering, hydrogeology, precipitation, climate change, hydrology, rain e rainfall; (C2) watersheds, simulation, geography, optimization, runoff, precipitation, water resources, models, 
statistical analysis, climate change, hydrogeology, floods, mathematical models, hydrology, rain, engineering e rainfall; (C3) storms, models, runoff, climate, water resources, earth sciences, floods, climate change, geography, statistical analysis, hydrogeology, engineering, precipitation, hydrology, mathematical models, rain e rainfall.

Após a aplicação dos filtros, foi feita a leitura dos títulos e resumos para selecionar quais trabalhos eram aderentes e o snowball sampling. Em seguida, a revisão da bibliografia foi desenvolvida a partir do número final de trabalhos aderentes.

Sabendo-se que o objeto de estudo do trabalho é a identificação dos métodos aplicados na determinação das curvas IDF, e tendo em vista as etapas para se calcular os parâmetros da curva IDF, foi realizado um levantamento de quais distribuiçóes probabilísticas, testes de aderência, fórmulas empíricas, métodos de otimização e avaliaçóes de ajuste, foram mais utilizados na elaboração dos artigos selecionados. Os resultados obtidos, por meio da aplicação do mapeamento previamente descrito e do levantamento realizado com base nos artigos aderentes, são apresentados na forma de tabelas.

\section{RESULTADOS E DISCUSSÃO}

Com base no mapeamento, foi elaborada a Tabela 1, que quantifica os resultados obtidos para todas as combinaçôes, contendo entre estes o número total de artigos iniciais e aderentes de cada uma e o total de artigos finais aderentes ao tema.

Tabela 1. Resultados obtidos no mapeamento.

\begin{tabular}{llll}
\hline \multirow{2}{*}{\multicolumn{1}{c}{ Etapas }} & \multicolumn{2}{c}{ Objeto de estudo: Curva IDF } \\
\cline { 2 - 4 } & & \multicolumn{2}{c}{ Expressóes-chave } \\
\cline { 2 - 4 } & Combinaçáo 1 & Combinaçáo 2 & Combinaçáo 3 \\
\hline Número inicial de artigos na mesma busca & 1385 & 239 & 284 \\
Número total de artigos após análise de aderência de títulos & 176 & 24 & 35 \\
Número de artigos após leitura dos resumos e sem repetiçôes na mesma busca & 42 & 7 & 10 \\
Artigos repetidos entre as buscas & & 17 \\
Artigos do Snowball sampling & & 6 \\
Total de artigos aderentes ao tema & 48 & \\
\hline
\end{tabular}

A partir dos resultados verifica-se que a combinaçáo "Intensity-duration-frequency" e "Estimt*" (C1) foi a que mais retornou resultados sobre o tema, foram $1385(72,59 \%)$ do total de artigos retornados pela busca, enquanto que as demais combinaçóes, "Intensity-duration-frequency" e "Optimization" (C2) e "Intensity-duration-frequency" e "Algorithm" (C3), retornaram 239 (12,53\%) e 284 (14,88\%), respectivamente.

Após a análise de aderência de títulos e resumos dos trabalhos, a C1 ainda continuou a totalizar o maior número de artigos aderentes, 42 artigos no total, que correspondem a $71,19 \%$, em relação a $7(11,86 \%)$ e 10 $(16,95 \%)$ das combinações C2 e C3, respectivamente. Feita a exclusão dos artigos repetidos (17 artigos no total) e realizado o snowball sampling (6 artigos) obteve-se um total de 48 artigos aderentes ao tema.

Em relação ao número de publicaçóes, em intervalos de 5 em 5 anos, empregando-se as três combinações de palavras-chave utilizadas na pesquisa até a data limite de 31 de dezembro de 2019, verifica-se que há um aumento do número de pesquisas a partir do ano de 2000, tendo em vista que nos períodos de 1985 a 1989, 1990 a 1994, 1995 a 1999 só há 1 artigo aderente por período. Esse número então triplica no período de 2000 a 2004 apresentando 3 artigos aderentes, dobra no período de 2005 a 2009 alcançando a marca de 6 artigos aderentes e aproximadamente triplica novamente apresentando 17 artigos aderentes no período de 2010 a 2014, praticamente se igualando no último intervalo, de 2015 a 2019, com 19 artigos aderentes.

Analisando-se de uma perspectiva continental, a América lidera com 50\% das publicaçóes, seguida pela Ásia, com $27,08 \%$, somando juntas cerca de $77 \%$ do total de artigos selecionados. Os demais 23\% dos trabalhos selecionados são compostos pela Europa, Oceania e África, tendo a última apresentado apenas 
um artigo aderente.

Em relação aos países, o Brasil é o país que mais apresenta publicaçóes aderentes, com um total de $39,58 \%$, seguido pela Turquia com $10,42 \%$ e pela Austrália com 8,33\% do total. Outros países apontados no mapeamento foram: Arábia Saudita e Canadá, com 6,25\% cada; Índia, Itália, Malásia e República Eslovaca, com 4,17\% cada; e Emirados Árabes, Estados Unidos, Grécia, México, República Checa e Zâmbia, com $2,08 \%$ cada. A grande porcentagem de publicaçóes brasileiras mostra uma grande relevância em relação ao tema no país e a importância de seu estudo.
Em relação aos periódicos onde esses artigos foram publicados (Tabela 2), verifica-se que a Revista Brasileira de Engenharia Agrícola e Ambiental e a Revista Água e Ambiente foram as que mais apresentaram publicaçóes, um total de 5 para cada. Quanto ao local de publicação, $37,50 \%$ dos artigos foram publicados em revistas nacionais, sendo que 55,5\% desses na Revista Brasileira de Engenharia Agrícola e Ambiental e na Revista Água e Ambiente. Em contrapartida, as revistas internacionais acumularam um total de $62,50 \%$ do total de publicaçôes, sendo que 13,33\% desses publicados no Journal of Hydrology.

Tabela 2. Quantitativo dos artigos obtidos no mapeamento por periódico.

\begin{tabular}{|c|c|}
\hline Periódicos & Número de Artigos Publicados \\
\hline Arabian Journal of Geosciences & 2 \\
\hline Canadian Journal of Civil Engineering & 1 \\
\hline Engenharia Agrícola & 2 \\
\hline Environmental Earth Sciences & 1 \\
\hline Estonian Journal of Earth Sciences & 1 \\
\hline Hydrological Processes & 3 \\
\hline Ingeniería Investigación y Tecnología & 1 \\
\hline International Journal of Climatology & 1 \\
\hline International Journal of Sustainable Built Environment & 1 \\
\hline Journal of Hydrologic Engineering & 2 \\
\hline Journal of Hydrology & 4 \\
\hline Journal of Hydrology and Hydromechanics & 1 \\
\hline Journal of Hydrology: Regional Studies & 1 \\
\hline Journal of King Saud University - Engineering Sciences & 2 \\
\hline KSCE Journal of Civil Engineering & 1 \\
\hline MATEC Web of Conferences & 1 \\
\hline Natural Hazards & 2 \\
\hline Pamukkale University Journal of Engineering Sciences & 1 \\
\hline Pesquisa Agropecuária Tropical & 2 \\
\hline Revista Ambiente \& Água & 5 \\
\hline Revista Brasileira de Engenharia Agrícola e Ambiental & 5 \\
\hline Revista Ciência Agronômica & 1 \\
\hline Revista Engenharia na Agricultura & 3 \\
\hline Slovak Journal of Civil Engineering & 1 \\
\hline SpringerPlus & 1 \\
\hline Stochastic Environmental Research and Risk Assessment & 1 \\
\hline Theoretical and Applied Climatology & 1 \\
\hline
\end{tabular}

$\mathrm{Na}$ Tabela 3 estão apresentadas as distribuiçóes período de retorno. A distribuição mais utilizada foi probabilísticas utilizadas na determinação da a de Gumbel (EV1), 68,75\% em relação ao total precipitação máxima associada a cada duração e de trabalhos analisados, seguida pela distribuição 
Generalizada de Valores Extremos (GEV) e pela distribuição Log-Person III, com 43,75\% e 41,67\%, respectivamente. Ressalta-se que os dados apresentados não possuem somatório igual a $100 \%$, tendo em vista que a maioria dos trabalhos analisados apesentam o uso de mais de um tipo de distribuição probabilística, de teste de aderência, de fórmula empírica, de método de otimização ou de avaliação de ajuste ou não apresentam um ou vários desses passos, pois focam seus estudos em uma dessas etapas.

O motivo de tal uso pode ser explicado por Oliveira et al. (2005), que afirmam que as distribuiçóes de valores extremos de grandezas hidrológicas ajustamse satisfatoriamente à distribuição de Gumbel. No entanto, como pode ser observado em Franco et al. (2014), Dar, Maqbool e Raazia (2016) e Mamoon e Rahman (2017), a distribuição de Gumbel nem sempre pode resultar na melhor opção, podendo ocorrer melhores ajustes advindos de outras distribuiçóes, como a distribuiçáo GEV e a distribuição de Pearson III para algumas regiôes do Catar, da Índia e do Brasil. Além disso, de acordo com AlHassoun (2011), o método Log Pearson III é supostamente mais preciso, pois explica a assimetria na distribuição de dados.

Contudo, algumas dessas distribuições necessitam da determinaçáo de coeficientes próprios para cada conjunto de dados. Assim, o método de obtenção dos coeficientes das distribuiçóes probabilísticas passa a ser um fator significativo, pois pode causar diferenças nos resultados de aderência. Franco et al. (2014) obtiveram em seu estudo por meio da distribuiçáo de probabilidades GEV o ajuste mais adequado utilizando o método dos momentos-L na estimativa dos coeficientes e a para a distribuição Gumbel aplicando-se a máxima verossimilhança.

Tabela 3. Distribuições probabilísticas utilizadas nos artigos.

\begin{tabular}{|c|c|}
\hline Distribuições Probabilísticas & Quantidade de Vezes Utilizadas \\
\hline Distribuição Exponencial & 2 \\
\hline Distribuição Generalizada de Fréchet & 2 \\
\hline Gamma Generalizado & 2 \\
\hline Gamma III (G3) & 2 \\
\hline Gamma II (G2) & 5 \\
\hline Generalização da Distribuição Logística (GLO) & 4 \\
\hline Generalizada de Valores Extremos (GEV) & 21 \\
\hline Gumbel (EV1) & 33 \\
\hline Kappa & 2 \\
\hline Log-Normal (LN) & 3 \\
\hline Log-Normal III (LN3) & 11 \\
\hline Log-Normal II (LN2) & 11 \\
\hline Log-Pearson (LP) & 1 \\
\hline Log-Pearson III (LP3) & 20 \\
\hline Pareto (GPD) & 7 \\
\hline Pearson & 2 \\
\hline Person III & 8 \\
\hline Wakeby & 1 \\
\hline Weibull & 4 \\
\hline
\end{tabular}

$\mathrm{Na}$ Tabela 4 pode-se verificar quais testes de aderência foram mais utilizados para se determinar a distribuição probabilística mais adequada. De acordo com Franco et al. (2014) os testes de aderência podem variar significativamente para cada distribuição probabilística aplicada, não sendo possível determinar qual teste é o mais eficiente. O teste mais utilizado foi o teste de Kolmogorv-Smirnov (KS) que apareceu em $39,58 \%$ dos artigos selecionados, seguido pelo método do Qui-Quadrado (X ${ }^{2}$ ), com 27,08\%. Se levado em consideraçáo o somatório da quantidade de vezes que cada teste foi aplicado, os testes de Kolmogorv-Smirnov 
(KS), Qui-Quadrado (X²) e Anderson Darling (AD) aparecem 40 vezes (num total de 56), o que representa $71,43 \%$.

O equacionamento das curvas IDF ocorre, em geral, de maneira empírica e assim como nos demais tópicos abordados, não existe uma única equaçáo capaz de se adequar a todos os tipos de chuva. Assim, esta pode variar de acordo com cada região, sendo necessários vários estudos a fim de se determinar qual a melhor equação empírica para o lugar em questão.

Tabela 4. Testes de aderência utilizados nos artigos para avaliar a distribuição probabilística mais aderente.

\begin{tabular}{cc}
\hline Testes de Aderência & Quantidade de Vezes Utilizados \\
\hline Anderson Darling (AD) & 8 \\
Bootstrapping & 1 \\
Coeficiente de Correlação (CC) & 1 \\
Critério Bayesiano de Schwarz (BIC) & 1 \\
Critério de Informaçáo de Akaike (AIC) & 1 \\
Erro Absoluto Máximo (MAE) & 2 \\
Erro Padrão de Estimativa (EPE) & 1 \\
Erro Relativo & 2 \\
Filliben & 19 \\
Kolmogorov-Smirnov (KS) & 13 \\
Qui-Quadrado (X') & 1 \\
Raiz do Erro Quadrático Médio (RMSE) & 1 \\
Raiz Relativa Erro Quadrático Médio (RRMSE) & 1 \\
Razão de Momentos-L & 3 \\
Teste Z & 1 \\
\hline
\end{tabular}

As Eq. 1, 2, 3, 4 e 5 foram utilizadas nos artigos aderentes. Nota-se que a quantidade de parâmetros ajustáveis em cada uma varia de 5 (Eq. 2) a 3 (Eq. 4). A Eq. 1 foi a mais utilizada nos trabalhos, um total de 21 vezes, o que representa $43,75 \%$, seguida da Eq. 5 , com $14,58 \%$.

$$
\begin{gathered}
i_{\max }=\frac{a \cdot T_{r}^{b}}{(t+c)^{d}} \\
i_{\max }=(a \cdot \ln t+b) \cdot\left(c \cdot t^{d}-e\right) \cdot h_{60,2} \\
i_{\max }=\frac{\left(a \cdot\left(T_{r}+b\right)^{c}\right)}{(t+d)^{b}} \\
i_{\max }=e \cdot\left(a+b \cdot T_{r}^{c}+d \cdot(\ln t)^{2}\right) \\
i_{\max }=\frac{a \times T_{r}^{b}}{t^{c}+d}
\end{gathered}
$$

Os métodos utilizados para determinar os parâmetros da curva IDF são escolhidos de acordo com as variáveis do problema e com a configuração da equação IDF utilizada. Devido a essa gama de metodologias, algumas delas podem apresentar uma melhor eficiência em relação à outra, obtendo ajustes melhores e com um menor custo computacional.

A Tabela 5 apresenta os métodos de otimização utilizados nos artigos, nela 20,83\% do total de trabalhos utilizaram Mínimos Quadrados e 16,67\% Gradação Reduzida Generalizada (GRG). No entanto, não se pode afirmar nada a respeito de qual método é mais preciso ou mais eficiente em termos computacionais, já que essas análises raramente foram realizadas. $\mathrm{Na}$ maior parte dos trabalhos, tais como os de Damé et al. (2014), Borges e Thebaldi (2016), Almeida, Reis e Mendonça (2017) e Silva et al. (2018), utilizou-se o GRG disponibilizado pela ferramenta Solver do software Excel ${ }^{\circ}$.

Outro detalhe importante, referente à análise dos métodos de otimização, é o uso de algoritmos heurísticos na determinação dos parâmetros da curva IDF. Pode-se perceber que apenas 2 do total de trabalhos utilizaram algoritmos heurísticos, o que representa penas 4,17\%. Os algoritmos heurísticos 
utilizados foram: o Algoritmo de Enxame de Partículas (PSO), no trabalho de Karahan (2012), e o Algoritmo Genético (AG), no trabalho realizado por Karahan, Ceylan e Ayvaz (2007).

Juntamente com a determinação dos parâmetros da curva IDF, são realizados testes para se verificar o ajuste da curva. O cálculo desse ajuste, como já mencionado anteriormente, reflete no quão próximo os dados reais estão dos dados estimados, ou seja, o quão adequado é o modelo proposto. Nos trabalhos analisados, pode-se observar que esse cálculo sempre é realizado.

Tabela 5. Métodos de otimização utilizados nos artigos

\begin{tabular}{cc}
\hline Métodos de Otimização & Quantidade de Vezes Utilizadas nos Artigos \\
\hline Algoritmo de Enxame de Partículas (PSO) & 1 \\
\hline Algoritmo Genético (AG) & 1 \\
\hline Gradação Reduzida Generalizada (GRG) & 8 \\
\hline Momentos-L & 1 \\
\hline Mínimos Quadrados & 10 \\
\hline Chen & 1 \\
\hline Regressão Não-linear de Gauss-Newton & 2 \\
\hline Sherman-Morrison & 1 \\
\hline Regressão Polinomial & 1 \\
\hline
\end{tabular}

Juntamente com a determinaçáo dos parâmetros da curva IDF, são realizados testes para se verificar o ajuste da curva. O cálculo desse ajuste, como já mencionado anteriormente, reflete no quáo próximo os dados reais estão dos dados estimados, ou seja, o quão adequado é o modelo proposto. Nos trabalhos analisados, pode-se observar que esse cálculo sempre é realizado.

$\mathrm{Na}$ Tabela 6, verifica-se que o coeficiente de determinação $\left(\mathrm{R}^{2}\right)$ foi o mais utilizado, apresentando um total de $25,00 \%$, em relação ao total de trabalhos, seguido pelo erro relativo, com 8,33\%. Esses dois coeficientes mais o índice de concordância de Wilmott (d), a raiz do erro quadrático médio (RMSE) e o coeficiente de determinação ajustado $\left(\mathrm{R}_{\mathrm{aj}}{ }^{2}\right)$, somam um total de 52,08\% de todos os cálculos realizados.

\section{CONLUSÃO}

1. Ocorreu um aumento significativo nos últimos 10 anos das publicaçôes envolvendo o estudo de curva IDF, chegando a quase quatro publicaçóes por ano;

2. A metodologia envolvendo o cálculo da curva IDF possui uma dinâmica sequenciada de etapas, no entanto há uma grande quantidade de maneiras para se executar cada passo;

3. A distribuição probabilística teórica mais utilizada é a de Gumbel e o teste de aderência mais utilizado é o de Kolmogorov-Smirnov;

4. O uso de algoritmos heurísticos para o cálculo dos parâmetros da curva IDF é pouco frequente quando comparado aos demais métodos.

\section{AGRADECIMENTOS}

À Fundação de Amparo à Pesquisa do Estado de Goiás pela bolsa de fomento.

\section{LITERATURA CITADA}

ALHASSOUN, S. A. Developing an empirical formulae to estimate rainfall intensity in Riyadh region.

Journal of King Saud University-Engineering

Sciences, Elsevier, v. 23, n. 2, p. 81-88, 2011.

ALMEIDA, K. N.; REIS, J. A. T.; MENDONÇA, A. S. F. Emprego dos métodos expeditos de Chow Gumbel e Bell para formulação de equações de chuvas intensas-uma avaliação de desempenho. Ambiente \& Água-An Interdisciplinary Journal of Applied Science, v. 12, n. 2, p. 203-214, 2017.

BORGES, G. M. R.; THEBALDI, M. S. Estimativa da precipitação máxima diária anual e equação de chuvas intensas para o município de Formiga, MG, Brasil. Revista Ambiente \& Água, v. 11, n. 4, p. 891902, 2016.

CARDOSO, C.; ULLMANN, M.; BERTOL, I. Análise de chuvas intensas a partir da desagregação 
Tabela 6: Métodos para avaliação de ajuste dos parâmetros determinados utilizados nos artigos

\begin{tabular}{|c|c|}
\hline Ajustes Calculados & Quantidade de Vezes Utilizadas nos Artigos \\
\hline Acurácia do Método de Chen & 1 \\
\hline Coeficiente de Correlação (R) & 2 \\
\hline Coeficiente de Desempenho (c) & 2 \\
\hline Coeficiente de Determinação $\left(\mathrm{R}^{2}\right)$ & 12 \\
\hline Coeficiente de Determinação Ajustado $\left(\mathrm{R}_{\mathrm{aj}}^{2}\right)$ & 3 \\
\hline Coeficiente de Eficiência Ajustado (E') & 2 \\
\hline Coeficiente de Eficiência de Nash-. Sutcliffe (COE) & 1 \\
\hline Coeficiente de Variação do Erro Quadrático Médio da Raiz $\left(\mathrm{CV}_{\mathrm{RMSE}}\right)$ & 1 \\
\hline Desvio Percentual Médio Absoluto (DPMA) & 1 \\
\hline Desvio Quadrático Médio(DQM) & 1 \\
\hline Erro Absoluto Máximo (MAE) & 2 \\
\hline Erro Absoluto Médio (EAM) & 1 \\
\hline Erro Padrão de Estimativa (EPE) & 1 \\
\hline Erro Padrão de Estimativa (EPE) & 1 \\
\hline Erro Quadrático Médio (MSE) & 2 \\
\hline Erro Quadrático Total & 1 \\
\hline Erro Relativo & 4 \\
\hline Índice de Concordância de Wilmott (d) & 3 \\
\hline Porcentagem Média da Diferença (D) & 1 \\
\hline Qui-Quadrado $\left(X^{2}\right)$ & 1 \\
\hline Raiz do erro quadrático médio (RMSE) & 3 \\
\hline Raiz Relativa do Erro Quadrático Médio (RRMSE) & 1 \\
\hline Soma dos Erros Quadrados & 1 \\
\hline Teste F de Variância $\left(\mathrm{F}_{\text {est }}\right)$ & 1 \\
\hline Teste T de Student & 1 \\
\hline Viés Relativo & 1 \\
\hline
\end{tabular}

das chuvas diárias de Lages e de Campos Novos (SC). Rio Grande do Sul, Brazil. Engenharia Agrícola, v. Revista Brasileira de Ciência do Solo, Sociedade 34, n. 4, p. 660-670, 2014.

Brasileira de Ciência do Solo, v. 22, n. 1, p. 131-140, 1998.

DAR, A. Q.; MAQBOOL, H.; RAAZIA, S. An empirical formula to estimate rainfall intensity in COMPANHIA DE TECNOLOGIA DE Kupwara region of Kashmir valley, J and K, India. SANEAMENTO AMBIENTAL. Drenagem Urbana: In: EDP SCIENCES. MATEC Web of Conferences. Manual de projeto. São Paulo, 1980. [S.1.], 2016. v. 57, p. 03-10.

COSTA, C.; STAUT, S.; ILHA, M. Projeto de sistemas prediais hidráulicos sanitários com BIM: mapeamento da literatura. In: .[S.1.: s.n.], 2014. p. 2760-2769.

DAMÉ, R. D. C.; TEIXEIRA-GANDRA, C. F.; VILLELA, F. A.; SANTOS, J. P. D.; WINKLER A. S. Analysis of the relationship intensity, duration, frequency of disaggregated daily rainfall in southern
FRANCO, Camila S.; MARQUES, Rosângela F. P. V.; OLIVEIRA, Alisson S.; OLIVEIRA, Luiz F. C. de. Distribuição de probabilidades para precipitação máxima diária na Bacia Hidrográfica do Rio Verde, Minas Gerais. Revista Brasileira de Engenharia Agrícola e Ambiental, SciELO Brasil, v. 18, n. 7, p. 735-741, 2014. 
KARAHAN, Halil. Determining rainfall-intensityduration-frequency relationship using Particle Swarm Optimization. KSCE Journal of Civil Engineering, v. 16, n. 4, p. 667-675, 2012.

KARAHAN, Halil; CEYLAN, Halim; AYVAZ, M. T. Predicting rainfall intensity using a genetic algorithm approach. Hydrological Processes: An International Journal, v. 21, n. 4, p. 470-475, 2007.

KITCHENHAM, B.; CHARTERS, S. Guidelines for performing systematic literature reviews in software engineering. Citeseer, 2007.

MAMOON, A. A.; RAHMAN, A. Selection of the best fit probability distribution in rainfall frequency analysis for Qatar. Natural hazards, Springer, v. 86, n. 1, p. 281-296, 2017.

OLIVEIRA, L. C.; CORTÊS, F. C.; SARMENTO, P. H. L.; WEHR, T. R.; BORGES, L. B.; GRIEBELER, N. P. Intensidade-duração-frequência de chuvas intensas para localidades no estado de goiás e distrito federal. Pesquisa Agropecuária Tropical, v. 35, p. 13-18, 2005.

PINTO, N. L. de S.; HOLTZ, A. C. T.; MARTINS, J. A.; GOMIDE, F. L. S. Hidrologia Básica. São Paulo: Blucher, 1976.

SILVA, J. B. L.; CÂNDIDO, F. A.; PIRES, L. C.; FRANÇA, L. C. J. NOTA TÉCNICA: EQUAÇÓES DE INTENSIDADE, DURAÇÃO E FREQUÊNCIA DE CHUVAS MÁXIMAS PARA O ESTADO DO RIO GRANDE DO NORTE, BRASIL. Revista Engenharia na Agricultura, v. 26, n. 2, p. 160-170, 2018. 